OPEN ACCESS

Edited by:

Oyewole Oyekoya,

Hunter College (CUNY), United States

Reviewed by:

Omar Janeh,

University of Hamburg, Germany

Allison Paige Anderson,

University of Colorado Boulder,

United States

${ }^{*}$ Correspondence:

Christoph Rockstroh

christoph.rockstroh@

psychologie.uni-freiburg.de

tThese authors have contributed equally to this work and share first authorship

Specialty section:

This article was submitted to Virtual Reality in Medicine,

a section of the journal

Frontiers in Virtual Reality

Received: 24 August 2020

Accepted: 27 October 2020

Published: 20 November 2020

Citation:

Rockstroh C, Blum J, Hardt V and

Göritz AS (2020) Design and

Evaluation of a Virtual Restorative Walk With Room-Scale Virtual Reality and

Impossible Spaces.

Front. Virtual Real. 1:598282.

doi: 10.3389/frvir.2020.598282

\section{Design and Evaluation of a Virtual Restorative Walk With Room-Scale Virtual Reality and Impossible Spaces}

\author{
Christoph Rockstroh ${ }^{\star \dagger}$, Johannes Blum ${ }^{\dagger}$, Véronique Hardt and Anja S. Göritz \\ Department of Occupational and Consumer Psychology, Albert-Ludwigs-Universität Freiburg, Freiburg, Germany
}

Nature walks are an effective and popular means to replenish fatigued mental resources. Alas, nature walks are not always accessible due to a lack of time or limited availability. We report on the design and pilot test of a room-scale virtual reality (VR) application that makes use of actual walking and impossible spaces to simulate dynamic restorative walks. We conducted a randomized controlled experiment in VR using a between-subjects design. Thirty-one participants went for an 8-min virtual walk either in a fixed virtual restorative environment (control condition) or in the proposed dynamic virtual restorative environment (treatment condition). The treatment condition with the proposed room-scale approach yielded increased user involvement, higher present moment awareness, increased perceived restorativeness of the experience, better subjective restoration, and an improved positive affect. Behavioral analysis showed that the proposed approach led to a reduced walking speed over the course of the walking exercise. The results suggest that room-scale VR in conjunction with virtual restorative environments and impossible spaces can be used to create sophisticated virtual restorative walks in confined spaces. Future research and development are needed to further establish the effects, to identify moderating and mediating factors and to investigate such VR applications in relevant real-world contexts.

Keywords: virtual reality, mental health, perceived restorativeness, attention restoration, walking, room-scale, nature walk

\section{INTRODUCTION}

\section{Restorative Nature Walks}

Nature walks are a prominent and effective way to take a break from stressful day-to-day routines and find relaxation as well as restoration. Numerous empirical studies have found that walks in natural settings such as parks or forests can successfully reduce symptoms of stress, improve mood and well-being, and restore mental resources (Pretty et al., 2005b; Marselle et al., 2014; Capaldi et al., 2015; Korpela et al., 2016; Lahart et al., 2019). Most often, the effect of nature walks is positively contrasted against walks in urban environments or indoor environments with human-made features and stimuli (Berman et al., 2008; Mayer et al., 2009; Roe and Aspinall, 2011).

Evidently, there are two factors that contribute to the restorative effects of nature walks, namely (1) being present in a natural environment and (2) moving about by means of walking. The restorative effects of nature environments have been extensively investigated. The Attention Restoration Theory (ART; Kaplan and Kaplan, 1989; Kaplan, 1995) posits that spending time in environments with restorative qualities allows for indirected effortless attention so that the mind 
can rest and replenish valuable but fatigued resources (directed attention fatigue; cf. Kaplan and Berman, 2010). The theory identifies four characteristics that make up a restorative environment. First, the environment needs to provide a feeling of being away. This can either happen through physical distancing, i.e., physically being in a remote location, or by psychological distancing, i.e., mentally detaching from day-to-day routines and everyday worries. The more the environment can support those processes the more restorative it is. Second, it needs to include elements that elicit soft fascination. It is crucial that the environment comprises stimuli which naturally draw the involuntary attention while allowing directed attentional processes to rest. Third, it needs to provide a high level of coherence and extent. The elements of the environment need to be perceived as connected in non-confusing ways and the environment as a whole encourages immersion and is large enough to allow for exploration. Fourth, it needs to have a high compatibility with the individual's preferences and goals, which is facilitated when there is an intrinsic motivation to perform enjoyable activities in that specific environment.

While these characteristics may be found in different kinds of environments, nature environments are deemed to fulfill them more likely or completely than other environments. The extensive space and the multitude of fascinating stimuli make nature environments draw involuntary attention. At the same time, they offer space for personal reflection, which fosters the restoration of fatigued mental resources. A range of empirical studies support this postulated effect of nature environments on well-being and restoration (Bowler et al., 2010; Berto, 2014; Ohly et al., 2016; Pritchard et al., 2020). Even brief views of natural environments can have a restorative effect ( $\mathrm{Li}$ and Sullivan, 2016). This effect seems to be more pronounced the more the nature environment is fully experienced (e.g., walking through the environment) instead of just observed (e.g., watching a video; Mayer et al., 2009; Roe and Aspinall, 2011).

The second factor that makes up a restorative walk is the actual walking. Traversing through a restorative environment has two benefits in terms of restoration and well-being. First, it introduces a mild form of physical activity, which can elicit a restorative effect and improve mood (Pretty et al., 2005b; Hug et al., 2008; Lahart et al., 2019). Walking is an available and easy to perform physical activity that forms a contrast to sedentary occupations (Pretty et al., 2005a). Regular physical activity in the form of leisure walks has been shown to improve well-being as well as several aspects of mental and physical health (Sakuragi and Sugiyama, 2006; Thompson Coon et al., 2011; Hanson and Jones, 2015; Gidlow et al., 2016). Second, walking can amplify the restorative qualities of the environment because it enables an active exploration of the surroundings. The experience of a larger space through walking can open up fascinating stimuli that draw the involuntary attention and increase the perceived coherence of the environment (Kaplan and Berman, 2010). The physical sensation of stepping into a restorative environment might also create a greater feeling of getting away from everyday worries. Performing different kinds of physical activity in nature environments has been found to have a greater potential for restoration and stress reduction than exercising in indoor or urban environments (Bodin and Hartig, 2003; Hug et al., 2009; Aspinall et al., 2015; Calogiuri et al., 2015, 2016).

All of the above may explain why restorative walks or nature walks are a prominent recreational activity in societies all over the world. But in our contemporary society, there are circumstances that might render it difficult to take recreational nature walks. For instance, an individual might live in an urban environment without green areas within a reasonable range or a lack of time to commute to said locations. Apart from that, an individual might be unable to leave their safe and secure space, for example a patient in a hospital, an elderly person unable to walk outside on their own, or an individual who is confined to indoor spaces due to imprisonment or due to a lockdown in a way as the Coronavirus pandemic made it necessary.

With increasing technological advances in computer simulations, a potential solution to the lack of practical availability of nature walks might be to simulate such experiences with modern virtual reality (VR) technology. By means of a sophisticated simulation, the restorative effects of nature walks could be made more accessible in situations in which they are otherwise unavailable. In the present paper, we propose and evaluate a VR application that makes use of mobile room-scale VR to offer an easy-to-use and always available surrogate of restorative walks. In the following, we first describe the components, design choices and advantages of the developed application and then report on a pilot study to gather data as to the feasibility of such an application.

\section{State-of-the-Art Virtual Reality}

When considering a technological solution for creating a virtual restorative environment (VRE), VR seems to be promising. Head-mounted VR offers a greater sensory fidelity than any other technology, a characteristic that is referred to as immersion (Slater and Wilbur, 1997). A high degree of immersion is important as it precedes a sense of presence, that is, the feeling of actually being inside a simulated environment (Cummings and Bailenson, 2016; Buttussi and Chittaro, 2017). The greater the sense of presence, the more the user can benefit from exposure to a virtual environment.

Contemporary VR headsets have improved greatly over the recent years, in terms of both fidelity and accessibility. The technology has become wireless and mobile by relying on internal instead of external processing and tracking units. This improves the overall availability and flexibility. Furthermore, commercial mobile headsets are capable of offering head tracking with six degrees of freedom (6-DoF; e.g., Oculus Quest headset, Pico Neo 2 headset). Both the user's head rotations and head translations while wearing the head-mounted display (HMD) are tracked in three dimensions (3D; cf. Janeh et al., 2019). This ensures a greater comfort in VR because the virtual movements are matched with the real-world movements. Even more importantly, in conjunction with the lack of wires, 6-DoF tracking allows for room-scale applications (Langbehn et al., 2017), that is, the user can freely move within the confines of their real room and thus move naturally inside the virtual room because all real-world movements are tracked and represented in the virtual space. Given the accessibility of mobile VR headsets 
and the importance of 6-DoF tracking, we chose to design and develop an application for such a mobile 6-DoF headset to offer high practicality. Notably, this comes with a number of challenges because the central processing unit (CPU) and graphics processing unit (GPU) are not as capable in a mobile headset as they are in a stationary computer. Given that VR is a processing-heavy technology in general, this needs to be kept in mind when designing the stimuli and user interaction.

\section{Virtual Restorative Environments}

Drawing from ART as described above, typical elements that can be found in nature appear to be a suitable setting for restorative walks. Therefore, when designing a virtual restorative walk, the computer-simulation of elements and stimuli resembling nature is the most straightforward approach. A number of experimental studies showed that simulated VREs can mimic the restorative effects of real-world nature environments (Annerstedt et al., 2013; Anderson et al., 2017; Chung et al., 2018; Laurent et al., 2019; Mattila et al., 2020). This seems to be especially true for sophisticated and immersive simulations, for example via HMDs (de Kort et al., 2006; Villani and Riva, 2012; Liszio et al., 2018; Blum et al., 2019; Rockstroh et al., 2019, 2020).

However, realistic nature simulations raise a concern from an accessibility point of view. When designing VREs for mobile VR headsets, realistic nature simulations with high resolution textures and an abundance of stimuli are not feasible. Such realistic simulations have a high processing demand which cannot be met by current mobile GPUs and CPUs without compression and reduction in detail. Given that this research paper focuses specifically on practicality and accessibility, we therefore chose to implement stylized variants of different natural elements and environments, which are less demanding in terms of computation. Notably, for an environment to meet the characteristics of restorativeness as described by ART (Kaplan and Kaplan, 1989; Kaplan, 1995), it does not have to be a realistic environment. Rather, the theory itself posits abstract requirements (i.e., being away, fascination, coherence, compatibility) that should possibly be fulfilled by a variety of environments that resemble nature. In the proposed application, we designed four different schemes of stylized VREs to offer variation (see Figure 1). Those VREs included stylized natural elements such as rocks, grass, flowers, and trees. Despite not comprising realistic stimuli, the design and layout of the VREs was guided by the layout of prototypical nature scenery in conjunction with the principles of ART.

Besides accessibility considerations, stylized environments are less likely to trigger specific associations to known stimuli and thus less likely to induce an uncanny valley effect, which describes the phenomenon that the more realistic a simulation becomes, the greater the dislike of this simulation can get (cf. Tinwell, 2014; McMahan et al., 2016). This would be detrimental in the context of restoration. Additionally, based on the lack of real-world associations and corresponding expectations, stylized stimuli allow for more creative freedom in designing the VRE. In an unrealistic environment, users are more open to unusual colors and shapes or novel arrangements of stimuli. Again, this makes it easier to design varying VREs for mobile VR.

\section{Locomotion and Impossible Spaces}

Besides a VRE, a restorative walking application needs to include a locomotion mechanic to simulate walking. There are a number of potential locomotion mechanics in VR. The most common one is artificial sliding locomotion with the help of a joystick or another user input. Whenever the user gives a certain type of input, he or she moves in the desired direction in the virtual world. While this type of locomotion allows for a maximum of freedom (i.e., all of the virtual world is easily accessible), it bears the greatest potential of simulator sickness due to the mismatch between virtual movement and real-world stillness (cf. Calogiuri et al., 2018; Saredakis et al., 2020). More importantly, it does not include the actual act of walking and would exclude the physical activity from the restorative walk. To our best understanding, a sophisticated simulation of nature walks must include a walking simulation that comprises the physical sensation and motor activity of actual walking. In the context of this paper, the use of additional equipment, like treadmills, is not investigated because the focus of our research approach lies on practicality and accessibility. We believe that an appropriate form of locomotion when simulating a restorative walk is to implement room-scale VR and precisely translate the user's real-world head position to the virtual world. As outlined above, modern mobile VR headsets make that possible. That way, the real-world sensation of walking through a restorative environment is accurately simulated in the virtual world with little risk of simulator sickness (cf. Saredakis et al., 2020) and a maximum of control over the locomotion. It is also an intuitive form of locomotion as it relies on real walking without the need for artificial user input or additional hardware and thus caters to the goal of high accessibility.

The room-scale approach, however, poses a challenge. In VR, it is easy to simulate extensive spaces that are larger than the real space that surrounds the user. But if the virtual movement is determined by real-world movement, the virtual space that can be traversed is restricted to the actual available real-world space. However, restorative walks typically include traversing extensive environments. A restriction of the virtual space to the available real-world space could reduce the restorative potential of the virtual walk by limiting the explorable extent of the environment and thus the abundance of stimuli that can be experienced from up close.

To ensure exploration of a large enough natural environment and thus increase the restorative quality of the experience while owing to the constraints of the limited available real-world space, the proposed virtual restorative walk makes use of so-called impossible spaces. Impossible spaces describe simulated rooms in which parts of the different spaces are overlapping and thus impossible in the real world (cf. Suma et al., 2012; Vasylevska et al., 2013). The overlap of said spaces is not directly visible to the user because it is occluded by objects that separate the spaces. Impossible spaces allow for a vast number of virtual environments in a finite amount of real-world space. That way, a simulated restorative walk can take place in a confined space and yet simulate an expansive VRE.

\section{Proof-of-Concept Study}

In the proposed restorative walking VR application, we implemented an impossible space layout that allows for a 

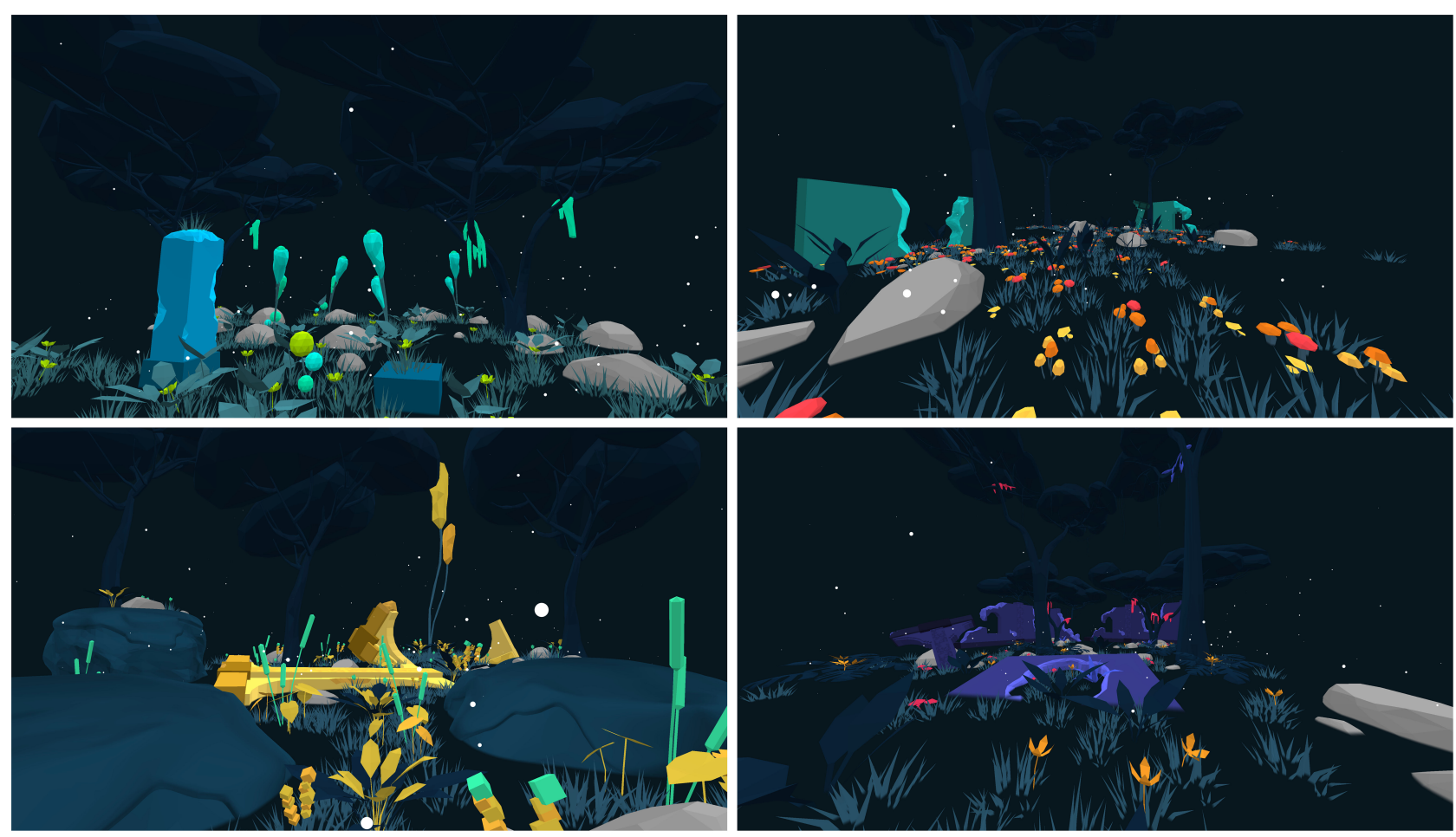

FIGURE 1 | Screenshots of some of the stylized nature environments.

repeated switching of the VREs (see Figure 2A). The user walks back and forth a defined path in the surrounding real room. The path is marked in the virtual world with small rocks indicating the borders of the walkable real world space and signaling the user when to turn around. We chose walking back and forth instead of walking in a circle because it limits the necessary space to one dimension and thus makes it even more accessible in confined spaces such as small furnished rooms or narrow hallways. Additionally, walking back and forth eliminates the need for pathfinding and thus allows the visual attention to be focused on the VRE. The virtual space is divided into two equally sized parts by a wall with a gate (see Figures 2B,C). While walking back and forth along the path, the user repeatedly traverses the gate. Each time the user walks through the gate, the gate briefly closes right behind the user, the (temporarily invisible) environment behind the wall is exchanged with a new environment and the gate immediately opens up again to reveal the new environment. The entire switching is completed within a second. The switching is unobtrusive because it is occluded and takes place behind the user who is currently still facing the other direction and walking to the end of the path. This combination of actual walking locomotion and impossible virtual spaces ensures that the user walks through a small portion of each VRE and that by means of walking the user can discover new surroundings and stimuli.

To investigate whether the proposed approach is feasible in a range of different real world environments, we piloted an experimental study. We compared the restorative walk with the dynamically changing environments to a control implementation with a fixed environment where the surroundings on each side of the wall stayed the same throughout the entire walking exercise. We assessed restoration and well-being as well as behavioral data of the respective walking exercise.

The environment-switching mechanic should offer a number of advantages over an implementation with a fixed environment in terms of the restorative potential of a room-scale virtual walk. First, while the user continuously walks back and forth inside the real space, he or she constantly traverses and explores new surroundings in the virtual space. Presumably, this makes it less apparent that the walking actually takes place in a highly limited space and only covers the same ground time and again. This should help the user feel more involved in the experience and be less aware of the potentially less restorative surrounding non-virtual space. We hypothesized the following:

Hypothesis $1\left(H_{1}\right)$ : A virtual restorative walk with dynamically changing VREs leads to a greater involvement than an implementation with a fixed environment.

Second, the greater abundance of fascinating stimuli should better capture the attention of the user. As a result, the user should have a greater present moment awareness with less distracting or unrelated thoughts that might dampen the restorative effect of the experience:

Hypothesis $2\left(\mathrm{H}_{2}\right)$ : A virtual restorative walk with dynamically changing VREs leads to a greater focus on the present moment than an implementation with a fixed environment. 

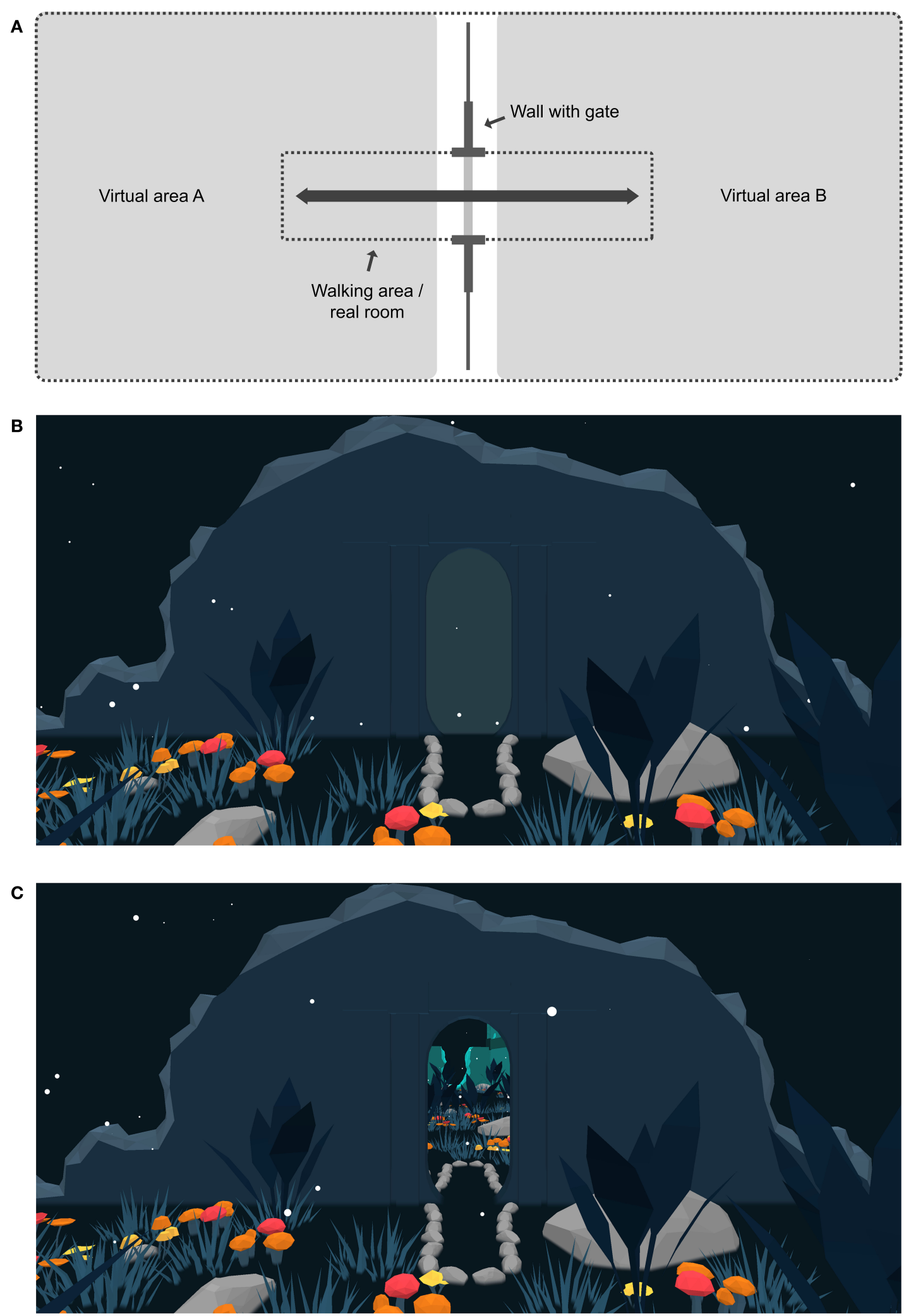

FIGURE 2 | (A) Layout of the virtual environment (view from top). The user walks back and forth within the walking area (dark double arrow). When the user steps into virtual area A, the gate closes and the environment in virtual area B is exchanged, and vice versa. (B) Screenshot of the separating wall with closed gate. The small rocks on the floor outline the area within which the participants are supposed to walk. (C) Screenshot of the separating wall with open gate. 
Third, instead of just walking back and forth inside a fixed environment, with impossible spaces the walking is a means to discover new surroundings. As argued above, the walking part in a restorative walk is not only done for the sake of physical exercise, but also for the sake of exploration. Based on this reasoning, we expected the following:

Hypothesis $3 a\left(H_{3 a}\right)$ : A virtual restorative walk with dynamically changing VREs has a greater restorative potential than an implementation with a fixed environment.

Hypothesis $3 b \quad\left(H_{3 \mathrm{~b}}\right)$ : A virtual restorative walk with dynamically changing VREs leads to a greater subjective restoration than an implementation with a fixed environment. Hypothesis $3 c\left(H_{3 c}\right)$ : A virtual restorative walk with dynamically changing VREs improves mood more than an implementation with a fixed environment.

Besides testing these hypotheses, we exploratively investigated whether the two virtual restorative walking implementations would affect the walking distance. The implementation of impossible spaces and the resulting variety of VREs might influence walking distance in competing ways. On the one hand, the dynamically changing VREs could result in curiosity to discover more, which would increase the walking speed and hence the total walking distance in a fixed time span. On the other hand, the greater density of novel stimuli could make the user take more time to take in each VRE, which would result in a decreased walking speed. Given those contradictory considerations, we assessed and analyzed the movement parameters in an explorative manner.

\section{METHOD}

\section{Participants and Design}

The study was conducted as a field experiment. The virtual walking experience as well as the control condition and all necessary assessments and information were included in a single Android application to work with an Oculus Quest VR headset. The app was made available to the public via the platform SideQuest (https://sidequestvr.com), which allows owners of the Oculus Quest headset to install third party apps on their device. The experiment was promoted via the SideQuest platform itself, as well as via different social media sites. There was no monetary reward for participation. All participants were given a license for personal use of the app for free after the experiment. This license enabled unlimited use without assessments and data collection.

In total, 31 participants (11 women) took part in the experiment. The average age was 37.6 years $(S D=9.8)$ with a range from 23 to 61 . The study was conducted as a randomized, controlled, between-subjects experiment with digitally obtained informed consent. The participants were randomly assigned to one out of two conditions, a virtual walk with a fixed environment (control group, $n=16$ ), or a virtual walk with the proposed dynamic environment switching mechanic (treatment group, $n$ $=15$ ). We measured involvement, present moment awareness, perceived restorativeness of the exercise, subjective restoration, mood as well as the actual distance walked. The experiment including random assignment was software-based without any experimenter interaction. The experiment was conducted in compliance with the APA ethics guidelines as well as all relevant local ethical requirements.

\section{Virtual Walking Exercise}

Participants in both conditions took part in an 8-min virtual walk, back and forth along a straight line with a length of $3.5 \mathrm{~m}$. In the treatment condition, we implemented the proposed impossible spaces, that is, a new stylized environment was instantiated whenever the participants stepped into the other half of the virtual space. To create variety, each environment was picked from one of four different schemes of environments (featuring different shapes and colors), each scheme including a range of similar environments. The color scheme from which the environments were drawn upon instantiation was changed every two minutes. The sequence of color schemes was random for each participant. In the control condition, there was no switching of environments. The environments on both sides of the gate were of the same scheme. For each participant in the control condition, one of the four schemes was randomly chosen. Both conditions received the same instructions (see Supplementary Material), which asked the participants to slowly walk back and forth along the path and take in the surroundings.

\section{Instruments}

We assessed both VR experience and meditation experience with a single-item each ("How experienced are you with VR / meditation?”) on a 5-point Likert scale (1 = not at all, $5=$ very well).

Involvement was assessed via the involvement subscale of the Igroup Presence Questionnaire (IPQ; Schubert et al., 2001). The subscale consists of four items, rated on 7-point Likert scales (sample item: "I was completely captivated by the virtual world").

Present moment awareness was measured via a self-framed item ("During the exercise, I felt closely connected to the present moment"), rated on a 7 -point Likert scale $(1=$ not at all, $7=$ completely).

The ascribed restorative quality of the respective walking exercise was measured with the Perceived Restorativeness Scale (PRS; Hartig et al., 1997). The PRS consists of the four subscales being away (two items; sample item: "Spending time here gives me a good break from my day-to-day routine"), fascination (six items; sample item: "The setting has fascinating qualities"), coherence (four items; sample item: "It is a confusing place"), and compatibility (five items; sample item: "I have a sense that I belong here"). For each item, participants rate their degree of approval on a 7 -point Likert scale $(1=$ not at all, $7=$ completely).

Subjective restoration was assessed with the Restorative Outcomes Scale (ROS; Korpela et al., 2008). The ROS comprises the three facets relaxation and calmness (three items; sample item: "After visiting this place, I now feel restored and relaxed"), attention restoration (one item; sample item: "My concentration and alertness clearly increased there"), and clearing one's thoughts (two items; sample item: "Visiting there was a way of clearing and clarifying my thoughts"), although all items are 
aggregated to form a single score for the entire scale. Each item is rated on a 7 -point Likert scale $(1=$ not at all, $7=$ totally). In contrast to the PRS, which asks participants to rate the perceived potential of a setting to elicit restoration in a variety of observers, the ROS addresses the actual momentary outcome of the experience in terms of subjective restoration.

Mood was measured via the short form of the Positive and Negative Affect Scale (PANAS-SF; Watson et al., 1988). It consists of two subscales for positive and negative affect with five items each. Participants rate to what extent the positive (sample item: "active") or negative (sample item: "nervous") adjective describes their current affect. Each item is rated on a 5-point Likert scale (1 $=$ not at all, $5=$ very much).

The walking distance was calculated by capturing all walkinginduced translations of the position of the VR headset on the $\mathrm{XZ}$-plane (i.e., horizontal translations only, excluding changes in the Y-axis due to unrelated up or down head movements) on a frame-by-frame basis.

\section{Procedure}

The entire experiment including all assessments and the walking exercise was performed within a dedicated VR application. At the outset, the app asked the participants to read and agree to an informed consent form. Next, the app checked whether the participant's playspace (marked and secured space in the real room tracked via the headset) was large enough (at least $0.75 \mathrm{~m} \times 4.0 \mathrm{~m})$ to take part in the experiment. In case of failure, participants were given the opportunity to rearrange their playspace and then continue with the experiment. Participants were guided toward a marked point in virtual space to ensure the correct starting position for the virtual walk to come. Next, participants answered the pre-assessment survey, which included demographic data and the PANAS-SF. After that, participants received and subsequently performed the 8 -min walking exercise in their randomly assigned experimental condition. After the exercise, participants answered the post-assessment survey, which included the PANAS-SF, the PRS, the ROS, the IPQ, and the item regarding present moment awareness. Lastly, the collected data were transmitted and participants were debriefed. From then on, all participants could replay the walking exercise of the treatment condition without any data collection.

\section{Data Analyses}

Given our experimental two-condition design with almost equal group sizes, random condition assignment and metric dependent variables, we followed the general recommendation (cf. Field, 2013) to test for potential between-subjects differences in outcome scores using analyses of variance (ANOVA) with a single fixed factor (experimental condition).

Prior to the analyses, we aggregated the corresponding items of each subscale of the IPQ, PRS, ROS, and PANAS-SF by computing the respective arithmetic mean. Each subscale of the PRS and the PANAS-SF were analyzed separately to provide more detailed insights as to the effects of the restorative walking exercise on the different facets of restorativeness and mood. To adjust for familywise error rates within the respective scales, the threshold for significance was Bonferroni-adjusted to $\alpha=0.0125$ for the PRS subscales and $\alpha=0.025$ for the PANAS-SF subscales. For all other instances of significance testing, the threshold was set to $\alpha=0.05$.

To check for potential confounds, we determined the between-condition differences as regards age, VR experience and meditation experience prior to the virtual walk with a one-way ANOVA. There was no effect of the condition on age, $F_{(1,29)}=$ $0.001, p=0.977, \eta_{\mathrm{p}}^{2}<0.001$, with similar values in the treatment group $(M=37.67, S D=10.04)$ and the control group $(M=$ $37.56, S D=9.84)$. There was no effect of the condition on VR experience, $F_{(1,29)}=0.034, p=0.855, \eta_{\mathrm{p}}^{2}=0.001$, with similarly high experience in the treatment group $(M=4.27, S D=1.22)$ and the control group $(M=4.19, S D=1.17)$. There was no effect of the condition on meditation experience, $F_{(1,29)}=0.102$, $p=$ $0.752, \eta_{\mathrm{p}}^{2}=0.004$, with similar values in the treatment group $(M=1.80, S D=1.01)$ and the control group $(M=1.69, S D=$ 0.95). Consequently, we did not include any covariates into our statistical models. The resulting single-factor ANOVA without any covariates is equivalent to a $t$-test for independent samples.

\section{RESULTS}

\section{Involvement}

To test whether the participants reported higher involvement in the treatment group as compared to the control $\left(H_{1}\right)$, we conducted a one-way ANOVA on the involvement subscale of the IPQ. The internal consistency of the subscale was Cronbach's $\alpha=0.92$. The ANOVA revealed an effect of the condition, $F_{(1,29)}$ $=16.677, p<0.001, \eta_{\mathrm{p}}^{2}=0.365$, with higher values in the treatment group $(M=5.75, S D=1.41)$ compared to the control group $(M=3.86, S D=1.16)$.

\section{Present Moment Awareness}

To test whether the participants reported higher present moment awareness after the treatment compared to the control $\left(\mathrm{H}_{2}\right)$, we conducted a one-way ANOVA on the self-framed item. The ANOVA revealed an effect of the condition, $F_{(1,29)}=8.840$, $p=0.006, \eta_{\mathrm{p}}^{2}=0.234$, with higher values in the treatment group $(M=5.87, S D=1.46)$ compared to the control group $(M=4.06, S D=1.88)$.

\section{Perceived Restorativeness}

To test for differences in participants' perceived restorativeness of the experience between the two conditions $\left(H_{3 a}\right.$; Figure 3$)$, we conducted a separate one-way ANOVA for each of the four subscales of the PRS. The internal consistencies of the subscales ranged from Cronbach's $\alpha=0.77$ (being away) to Cronbach's $\alpha=$ 0.95 (fascination and compatibility). The ANOVA on being away showed an effect of the condition, $F_{(1,29)}=7.679, p=0.010, \eta_{\mathrm{p}}^{2}=$ 0.209 , with higher values in the treatment group $(M=5.83, S D$ $=1.50)$ compared to the control group $(M=4.53, S D=1.10)$. The ANOVA on fascination revealed an effect of the condition, $F_{(1,29)}=17.738, p<0.001, \eta_{p}^{2}=0.380$, with higher fascination in the treatment group $(M=5.83, S D=1.12)$ compared to the control group $(M=3.94, S D=1.36)$.

There was no effect of the condition on coherence, $F_{(1,29)}=$ 3.904, $p=0.058, \eta_{\mathrm{p}}^{2}=0.119$, with comparable values in the 


\section{- Control घ Treatment}

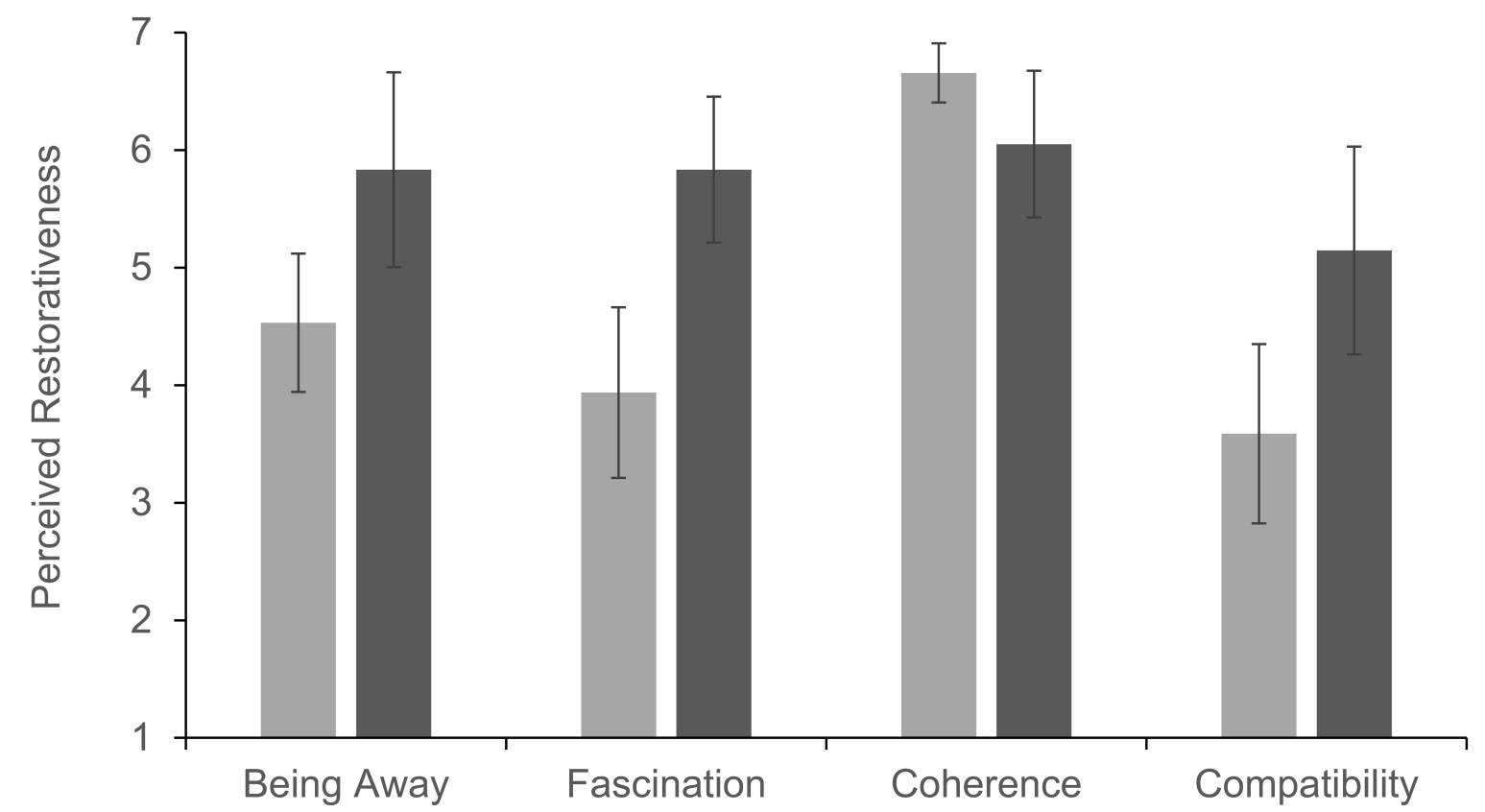

FIGURE 3 | Perceived restorativeness by subscale by condition. Error bars represent 95\% confidence intervals (95\% Cl).

treatment group $(M=6.05, S D=1.13)$ and the control group $(M=6.66, S D=0.47)$. The ANOVA on compatibility showed an effect of the condition, $F_{(1,29)}=8.230, p=0.008, \eta_{\mathrm{p}}^{2}=0.221$, with higher values in the treatment group $(M=5.15, S D=1.60)$ compared to the control group $(M=3.59, S D=1.43)$.

\section{Subjective Restoration}

To test whether the participants felt more restored after the treatment compared to the control $\left(H_{3 b}\right.$; Figure 4$)$, we conducted a one-way ANOVA. The internal consistency of the ROS was Cronbach's $\alpha=0.97$. The ANOVA revealed an effect of the condition, $F_{(1,29)}=6.270, p=0.018, \eta_{\mathrm{p}}^{2}=0.178$, with higher subjective restoration in the treatment group $(M=5.53, S D=$ $1.54)$ compared to the control group $(M=4.18, S D=1.48)$.

\section{Mood}

To test for different changes in mood from pre- to post-exercise between the two conditions $\left(H_{3 c}\right)$, we conducted two separate repeated-measures ANOVA for positive (Figure 5) and negative affect (Figure 6). The internal consistencies of the two subscales of the PANAS-SF ranged from Cronbach's $\alpha=0.78$ (PANAS-PA, pre-exercise) to Cronbach's $\alpha=0.94$ (PANAS-NA, pre-exercise). The ANOVA on positive affect showed a Time $\times$ Condition interaction, $F_{(1,29)}=18.813, p<0.001, \eta_{\mathrm{p}}^{2}=0.393$. The increase in positive affect was stronger in the treatment group $\left(\Delta_{\text {mean }}=\right.$ $1.43)$ compared to the control group $\left(\Delta_{\text {mean }}=0.13\right)$. There was no Time $\times$ Condition interaction on negative affect, $F_{(1,29)}<$ 0.001, $p=0.992, \eta_{\mathrm{p}}^{2}<0.001$. However, there was an effect of Time on negative affect, $F_{(1,29)}=26.876, p<0.001, \eta_{\mathrm{p}}^{2}=0.481$, with negative affect decreasing from pre-exercise $(M=1.75, S D$ $=0.95)$ to post-exercise $(M=1.33, S D=0.73)$.

\section{Distance Walked}

To test for differences in the distance walked during the exercise we conducted a one-way ANOVA (Figure 7). The ANOVA revealed an effect of the condition, $F_{(1,29)}=7.140, p=0.012$, $\eta_{\mathrm{p}}^{2}=0.198$, with a longer distance walked in the control group $(M=182.52 \mathrm{~m}, S D=46.99 \mathrm{~m})$ than in the treatment group $(M=143.05 \mathrm{~m}, S D=33.67 \mathrm{~m})$.

\section{DISCUSSION}

This study explored the feasibility of a novel room-scale VR approach to simulated restorative walks. It investigated whether a simulated virtual walk with dynamic VREs via impossible spaces has advantages over an implementation with a fixed environment in terms of involvement, present moment awareness, perceived restorativeness, subjective restoration, and mood. To explore behavioral implications, we analyzed the effect on the distance walked during the exercise.

The results are encouraging with regard to the novel roomscale VR approach to simulated restorative walks. Given that the study was conducted in the field with a range of unknown room layouts, the study provides an empirical proof-of-concept of the proposed approach in natural (i.e., out-of-lab) contexts. Specifically, as regards user involvement $\left(H_{1}\right)$, the results favor the implementation with dynamic VREs. As regards $\mathrm{H}_{2}$, the participants in the treatment condition had a greater 


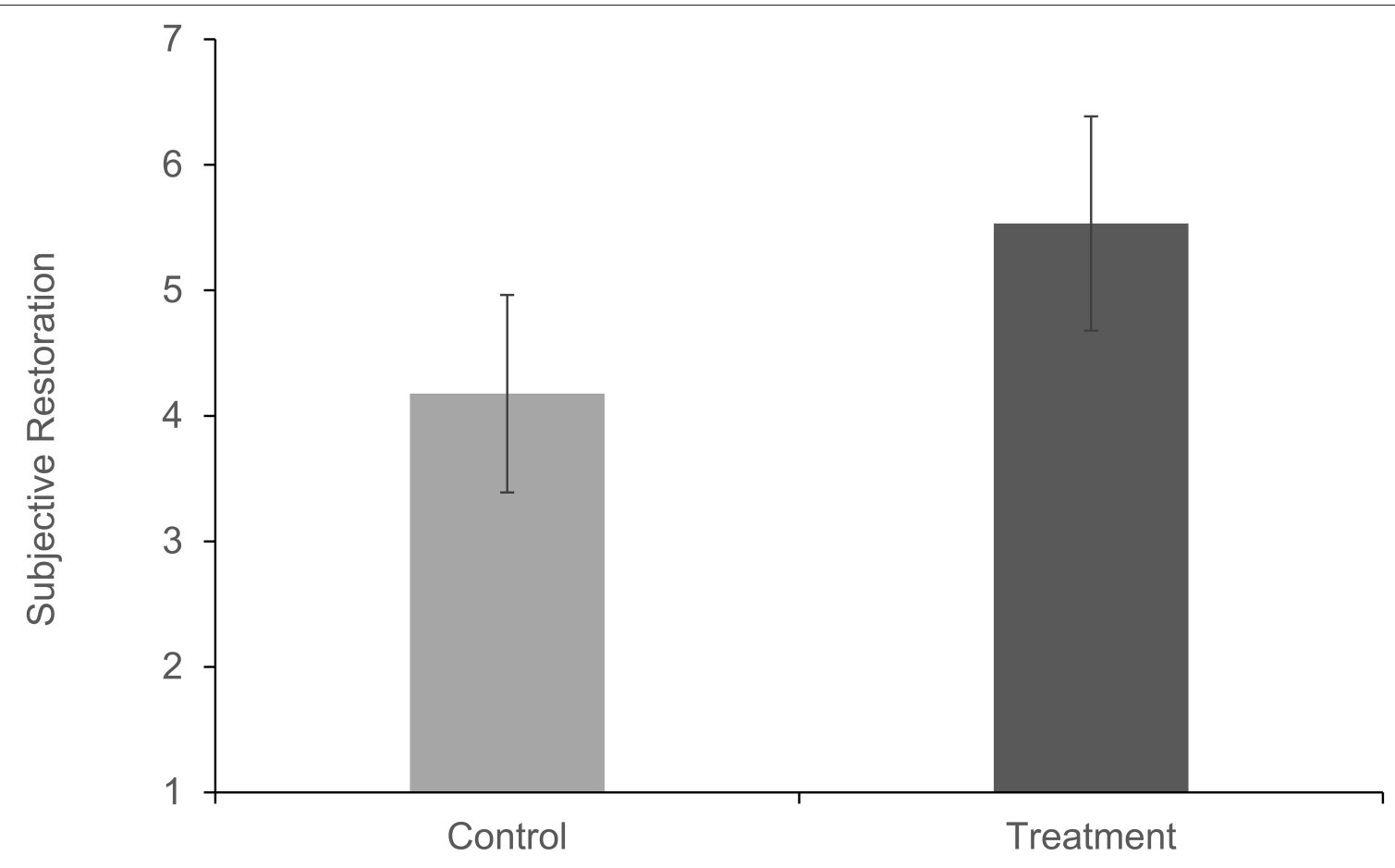

FIGURE 4 | Subjective restoration by condition. Error bars represent 95\% Cl.

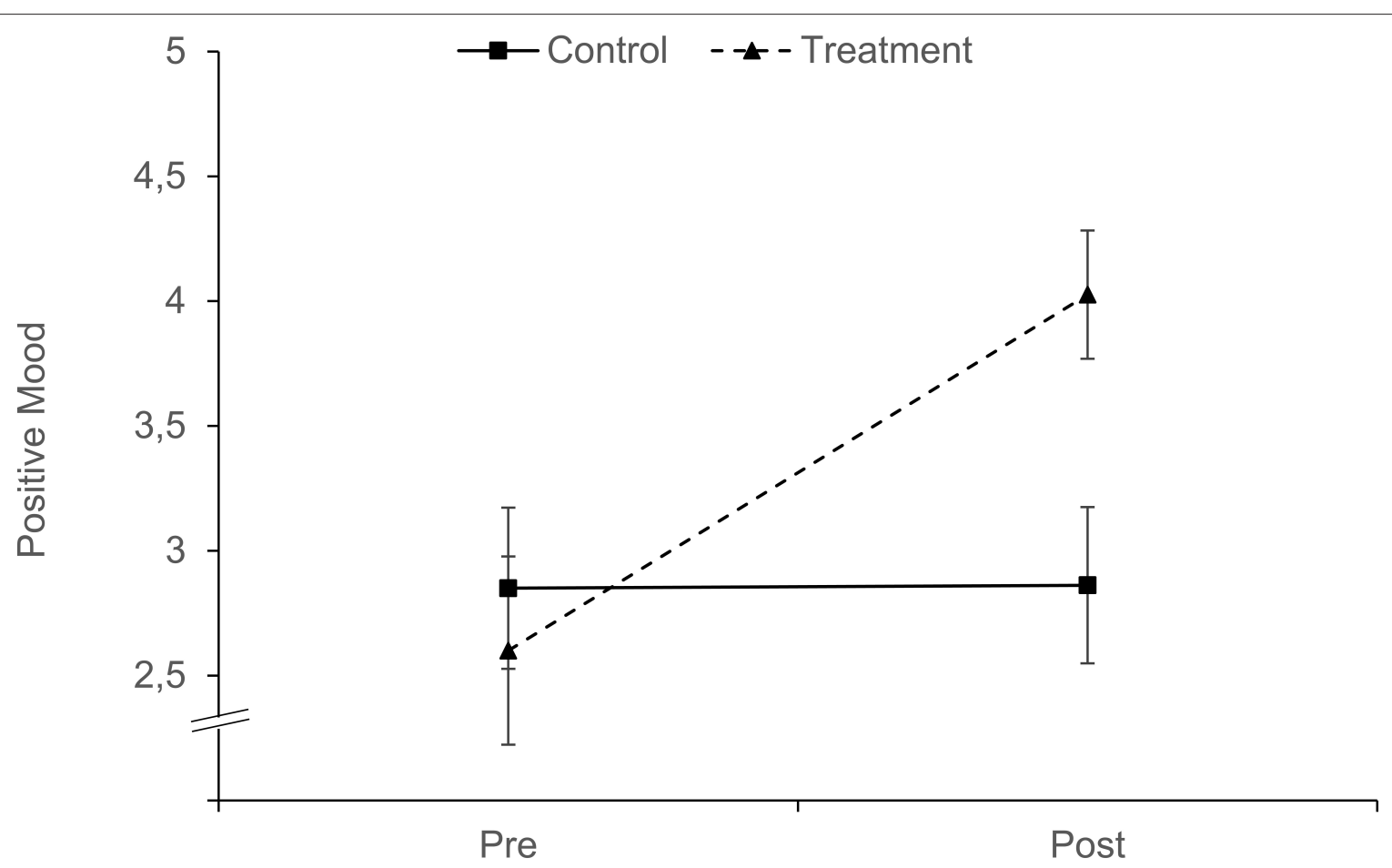

FIGURE 5 | Positive mood by condition by time point. Error bars represent 95\% Cl.

focus on the present moment. Capturing the user's focus is necessary in a restorative experience in which the surrounding stimuli themselves are supposed to elicit the desired restorative effect. Apart from the potential restorative effects, the greater possibility of exploration in the treatment condition offered more diversion and created a greater engagement than the supposedly 


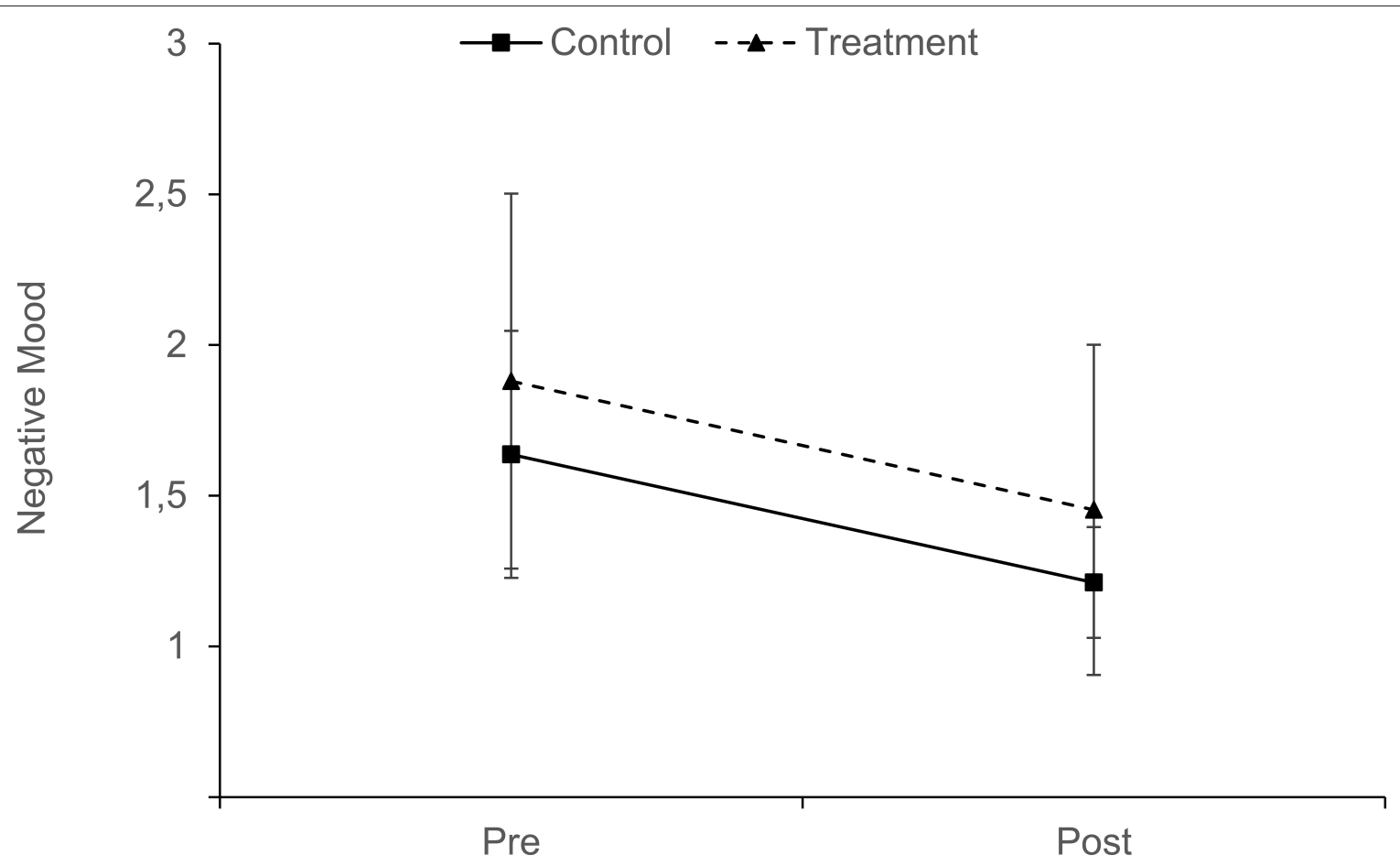

FIGURE 6 | Negative mood by condition by time point. Error bars represent 95\% Cl.

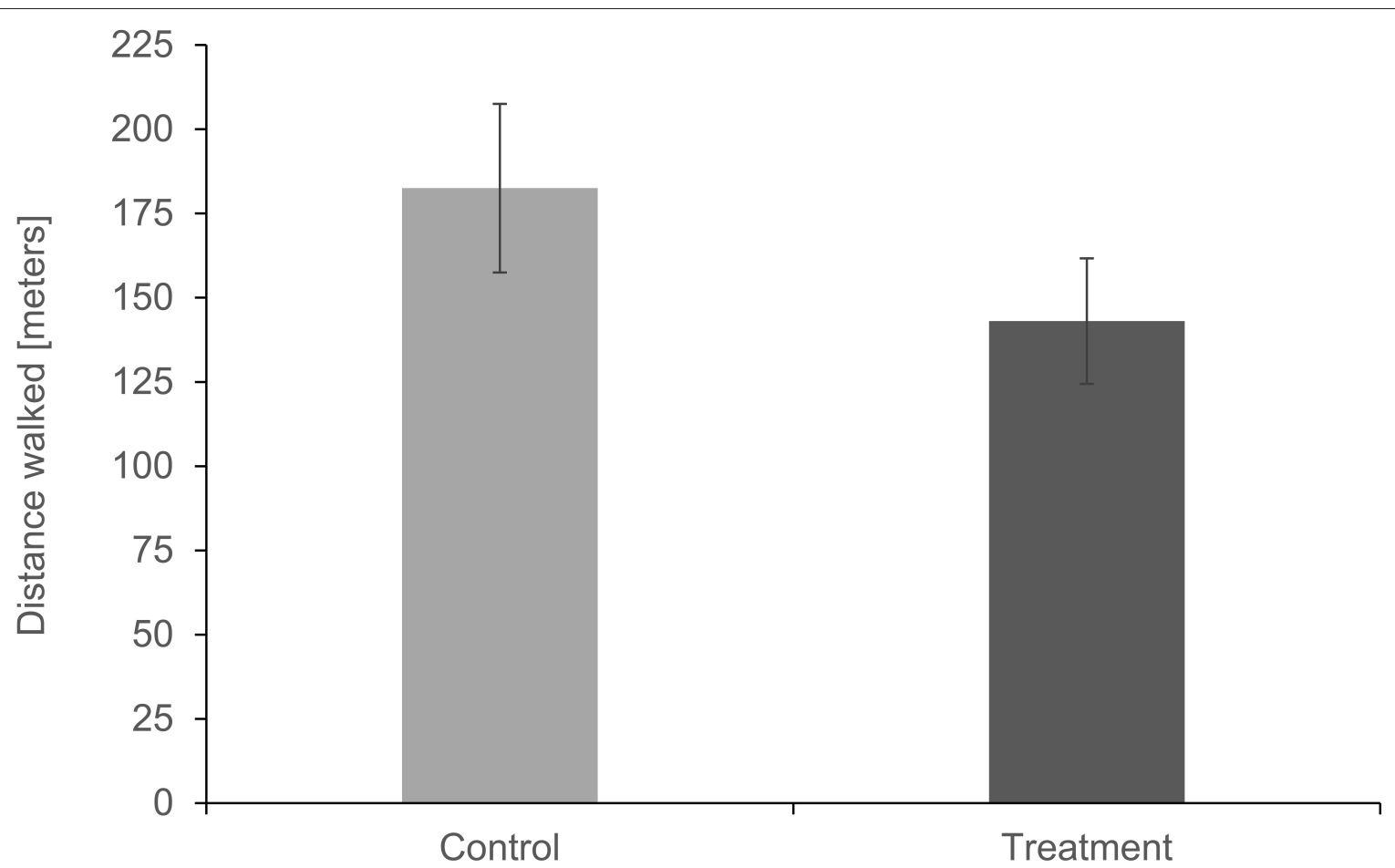

FIGURE 7 | Distance walked by condition. Error bars represent 95\% Cl. 
monotonous control condition which seems to have comprised a risk of attentional drift and rumination.

Furthermore, perceived restorativeness of the experience $\left(H_{3 \mathrm{a}}\right)$ and subjective restoration after the experience $\left(H_{3 \mathrm{~b}}\right)$ were rated higher in the treatment condition. Therein, participants reported greater values of being away, fascination and compatibility, which are indicative of a larger restorative potential. There was no effect of the condition on the coherence subscale. While the treatment condition did, as a matter of fact, offer more varying environments and thus had a larger explorable extent, this does not seem to have helped create a more coherent experience. A reason could be that the treatment condition comprised all four different environment schemes in terms of shapes and colors one after the other while the control condition only comprised one of those schemes. Moreover, the environment switching mechanic via impossible spaces might have reduced the feeling of coherence. However, coherence was at the high end of the scale in both conditions, which underlines the conclusion that the proposed dynamic restorative walking approach did not diminish the overall high degree of coherence. Nevertheless, the coherence facet of restorativeness should be investigated more closely in future research.

In terms of mood $\left(H_{3 c}\right)$, participants in the treatment condition reported higher increases in positive mood from pre- to post-exercise. Thus, the treatment condition with its higher restorative effects elicited more positive feelings than the control condition. Contrary to expectation, this difference was not present in the negative affect. Undergoing any condition of the exercise reduced negative affect down to the low end of the scale. This is a bottom effect, which might account for the fact that the reduction of bad mood was not larger in the treatment condition than in the control condition: In both conditions there was hardly any bad mood left after the exercise, leaving no room for the treatment condition to reduce bad mood even more.

Lastly, the study yielded interesting results as regards the distance walked. On average, participants in the treatment condition covered 143 meters, while participants in the control condition walked 183 meters. Over the course of the 8-min exercise, this translates to an average speed of $1.07 \mathrm{kph}$ in the treatment condition and $1.37 \mathrm{kph}$ in the control condition. Both velocities amount to very slow walking. In part, this low speed could be due to the fact that participants walked back and forth and, therefore, turned around every once in a while. Our result ties in with research on gait and velocity in VR: Velocity is reduced in room-scale VR compared to an otherwise identical real walking exercise (e.g., Janeh et al., 2019). The corroboration of the literature in this study's finding implies that our novel virtual restorative walking implementation is adequate if low walking speeds are acceptable or even desired. For higher targeted walking speeds, ours does not seem to be a useful implementation. Comparing the two conditions in this study, participants in the treatment condition walked even more slowly; they had more environments to explore than participants in the control condition who experienced the same environment again and again.

\section{LIMITATIONS AND FUTURE RESEARCH}

Despite the successful proof-of-concept, this study's limitations need to be considered and should be addressed in future research. First, the study included a small sample of 31 participants. While most effect sizes were large enough to test significant in spite of low statistical power, a larger sample size would be desirable. Additionally, participants with varying health backgrounds and varying experience with restorative walks and VR are necessary to extrapolate the results to a wider public. While experienced VR users are favorable in terms of ruling out novelty effects of the technology as well as technical issues with the operation of an HMD, the results cannot be generalized to inexperienced users. Furthermore, given that we targeted experienced VR users and implemented a natural locomotion scheme, we did not assess simulator sickness. However, this might have been an issue and would need to be addressed in future research. Second, due to the field setting, the real rooms were uncontrolled. However, given that we assigned participants to conditions at random, the low degree of control is unlikely to pose a threat to internal validity. While it is a strength that our study was conducted in the field and hence the robustness of its findings could be established, running the study in the lab would be useful for fine-tuning the paradigm to maximize its desired effects. Third, the study did not include objective physiological parameters of restoration that would support the self-reported evidence in this study. While the field setting creates a case for the real-world feasibility of the novel approach, the study should be repeated in a controlled lab setting with the inclusion of physiological measures such as heart rate, heart rate variability or electrodermal activity. Fourth, while the baseline mood was comparable in the two conditions, baseline negative affect was low and thus unlikely to be much reduced by the virtual restorative walks. To prevent such a bottom effect, a study that includes a stress induction prior to the treatment might prove useful. Moreover, such a stress induction could clarify whether the proposed procedure is effective as an acute stress reduction treatment.

Apart from suggestions for future research that derive from the limitations of the present study, we also suggest to look into the following. Future research should further disentangle the restorative effects of nature exposure and walking as well as their combination. Studies should investigate whether stylized vs. realistic nature environments differ in their restorative potential. Furthermore, different variants of environmental switching mechanics could be compared to the one implemented in this paper. In addition, the spatial layout for the room-scale tracking needs to be looked into. For instance, walking in a circle instead of walking back and forth could create a stronger feeling of continuity and thus coherence. It needs to be tested whether this potential effect outperforms the potentially distracting requirement for navigation as well as the need for larger realworld spaces that come with curves instead of simple linear paths. Lastly, different playspace sizes should be compared to determine the optimal and the minimal necessary playspace for the simulation of a virtual walk. 


\section{CONCLUSION}

The present paper reported on the design and evaluation of a room-scale VR application with impossible spaces to offer a sophisticated and yet low-threshold simulation of a restorative walk. We conducted a randomized controlled experiment as a proof-of-concept. The results show positive effects of the virtual restorative walk with impossible spaces on user involvement, present moment awareness, perceived restorativeness, subjective restoration and positive affect. Walking speed during the restorative walk was lower in the dynamic impossible spaces than in the fixed control environment. Future research studies need to investigate the proposed virtual walk in different contexts and determine the optimal real-world space for an effective virtual restorative walk.

\section{DATA AVAILABILITY STATEMENT}

The raw data supporting the conclusions of this article will be made available by the authors upon request.

\section{ETHICS STATEMENT}

Ethical review and approval was not required for the study on human participants in accordance with the local legislation and

\section{REFERENCES}

Anderson, A. P., Mayer, M. D., Fellows, A. M., Cowan, D. R., Hegel, M. T., and Buckey, J. C. (2017). Relaxation with immersive natural scenes presented using virtual reality. Aerosp. Med. Hum. Perform. 88, 520-526. doi: 10.3357/AMHP.4747. 2017

Annerstedt, M., Jönsson, P., Wallergård, M., Johansson, G., Karlson, B., Grahn, P., et al. (2013). Inducing physiological stress recovery with sounds of nature in a virtual reality forest - Results from a pilot study. Physiol. Behav. 118, 240-250. doi: 10.1016/j.physbeh.2013.05.023

Aspinall, P., Mavros, P., Coyne, R., and Roe, J. (2015). The urban brain: analysing outdoor physical activity with mobile EEG. Br. J. Sports Med. 49, 272-276. doi: 10.1136/bjsports-2012-091877

Berman, M., Jonides, J., and Kaplan, S. (2008). The cognitive benefits of interacting with nature. Psychol. Sci. 19, 1207-1212. doi: 10.1111/j.1467-9280.2008.02225.x

Berto, R. (2014). The role of nature in coping with psycho-physiological stress: A literature review on restorativeness. Behav. Sci. 4, 394-409. doi: $10.3390 /$ bs4040394

Blum, J., Rockstroh, C., and Göritz, A. S. (2019). Heart rate variability biofeedback based on slow-paced breathing with immersive virtual reality nature scenery. Front. Psychol. 10:2172. doi: 10.3389/fpsyg.2019.02172

Bodin, M., and Hartig, T. (2003). Does the outdoor environment matter for psychological restoration gained through running? Psychol. Sport Exerc. 4, 141-153. doi: 10.1016/S1469-0292(01)00038-3

Bowler, D. E., Buyung-Ali, L. M., Knight, T. M., and Pullin, A. S. (2010). A systematic review of evidence for the added benefits to health of exposure to natural environments. BMC Public Health 10:456. doi: 10.1186/1471-2458-10-456

Buttussi, F., and Chittaro, L. (2017). Effects of different types of virtual reality display on presence and learning in a safety training scenario. IEEE Trans. Visualiz. Comp. Graph. 24, 1063-1076. doi: 10.1109/TVCG.2017.2653117

Calogiuri, G., Evensen, K., Weydahl, A., Andersson, K., Patil, G., Ihlebæk, C., et al. (2016). Green exercise as a workplace intervention to reduce job stress. Results from a pilot study. Work. 53, 99-111. doi: 10.3233/WOR-152219 institutional requirements. The patients/participants provided their written informed consent to participate in this study.

\section{AUTHOR CONTRIBUTIONS}

$\mathrm{CR}, \mathrm{JB}, \mathrm{VH}$, and AG developed the study concept and design. $\mathrm{CR}, \mathrm{JB}$, and $\mathrm{VH}$ designed the virtual environment, collected the data and performed the data analyses. CR and JB programmed the experiment and drafted an initial version of the manuscript. $\mathrm{VH}$ and AG provided revisions. All authors approved the final version of the manuscript for submission. $\mathrm{CR}$ and JB contributed equally to this work and share first authorship.

\section{FUNDING}

The article processing charge was funded by the BadenWuerttemberg Ministry of Science, Research and Art and the University of Freiburg in the funding programme Open Access Publishing.

\section{SUPPLEMENTARY MATERIAL}

The Supplementary Material for this article can be found online at: https://www.frontiersin.org/articles/10.3389/frvir. 2020.598282/full\#supplementary-material

Calogiuri, G., Litleskare, S., Fagerheim, K. A., Rydgren, T. L., Brambilla, E., and Thurston, M. (2018). Experiencing nature through immersive virtual environments: Environmental perceptions, physical engagement, and affective responses during a simulated nature walk. Front. Psychol. 8:2321. doi: $10.3389 /$ fpsyg.2017.02321

Calogiuri, G., Nordtug, H., and Weydahl, A. (2015). The potential of using exercise in nature as an intervention to enhance exercise behavior: Results from a pilot study. Percept. Motor Skills 121, 350-370. doi: 10.2466\%2F06.PMS.121c17x0

Capaldi, C. A., Passmore, H. A., Nisbet, E. K., Zelenski, J. M., and Dopko, R. L. (2015). Flourishing in nature: a review of the benefits of connecting with nature and its application as a wellbeing intervention. Int. J. Wellbeing 5, 1-16. doi: 10.5502/ijw.v5i4.449

Chung, K., Lee, D., and Park, J. Y. (2018). Involuntary attention restoration during exposure to mobile-based 360 virtual nature in healthy adults with different levels of restorative experience: event-related potential study. J. Med. Int. Res. 20:e11152. doi: 10.2196/11152

Cummings, J. J., and Bailenson, J. N. (2016). How immersive is enough? A metaanalysis of the effect of immersive technology on user presence. Media Psychol. 19, 272-309. doi: 10.1080/15213269.2015.1015740

de Kort, Y. A., Meijnders, A. L., Sponselee, A. A., and IJsselsteijn, W. A. (2006). What's wrong with virtual trees? Restoring from stress in a mediated environment. J. Environ. Psychol. 26, 309-320. doi: 10.1016/j.jenvp.2006.09.001

Field, A. (2013). Discovering Statistics Using IBM SPSS Statistics (4th edition). London: Sage

Gidlow, C. J., Jones, M. V., Hurst, G., Masterson, D., Clark-Carter, D., Tarvainen, M. P., et al. (2016). Where to put your best foot forward: Psycho- physiological responses to walking in natural and urban environments. J. Environ. Psychol. 45, 22-29. doi: 10.1016/j.jenvp.2015.11.003

Hanson, S., and Jones, A. (2015). Is there evidence that walking groups have health benefits? A systematic review and meta-analysis. Br. J. Sports Med. 49, 710-715. doi: 10.1136/bjsports-2014-094157

Hartig, T., Korpela, K., Evans, G. W., and Gärling, T. (1997). A measure of restorative quality in environments. Scand. Hous. Plann. Res. 14, 175-194. doi: 10.1080/02815739708730435 
Hug, S. M., Hansmann, R., Monn, C., Krütli, P., and Seeland, K. (2008). Restorative effects of physical activity in forests and indoor settings. Int. J. Fitness 4, 25-38.

Hug, S. M., Hartig, T., Hansmann, R., Seeland, K., and Hornung, R. (2009). Restorative qualities of indoor and outdoor exercise settings as predictors of exercise frequency. Health Place 15, 971-980. doi: 10.1016/j.healthplace.2009.03.002

Janeh, O., Katzakis, N., Tong, J., and Steinicke, F. (2019). "Infinity walk in VR: effects of cognitive load on velocity during continuous long-distance walking," in Proceedings of the 2019 ACM Symposium on Applied Perception (Barcelona: Association for Computing Machinery), 1-9. doi: 10.1145/3343036.3343119

Kaplan, S. (1995). The restorative benefits of nature: toward an integrative framework. J. Environ. Psychol. 15, 169-182. doi: 10.1016/0272-4944(95)90001-2

Kaplan, S., and Berman, M. G. (2010). Directed attention as a common resource for executive functioning and self-regulation. Persp. Psychol. 5, 43-57. doi: $10.1177 / 1745691609356784$

Kaplan, S., and Kaplan, R. (1989). The Experience of Nature: A Psychological Perspective. Cambridge: Cambridge University Press

Korpela, K. M., Stengård, E., and Jussila, P. (2016). Nature walks as a part of therapeutic intervention for depression. Ecopsychology 8, 8-15. doi: $10.1089 /$ eco.2015.0070

Korpela, K. M., Ylén, M., Tyrväinen, L., and Silvennoinen, H. (2008). Determinants of restorative experiences in everyday favorite places. Health Place 14, 636-652. doi: 10.1016/j.healthplace.2007.10.008

Lahart, I., Darcy, P., Gidlow, C., and Calogiuri, G. (2019). The effects of green exercise on physical and mental wellbeing: a systematic review. Int. J. Environ. Res. Public Health 16:1352. doi: 10.3390/ijerph16081352

Langbehn, E., Lubos, P., Bruder, G., and Steinicke, F. (2017). Bending the curve: Sensitivity to bending of curved paths and application in room-scale VR. IEEE Trans. Visual. Comp. Graph. 23, 1389-1398. doi: 10.1109/TVCG.2017.2657220

Laurent, H. K., LaValle, S. M., Mimnaugh, K. J., and Browning, M. H. (2019). Can simulated nature support health? Comparing short, single-doses of 360-degree nature videos in virtual reality with the outdoors. Front. Psychol. 10:2667. doi: 10.3389/fpsyg.2019.02667

Li, D., and Sullivan, W. C. (2016). Impact of views to school landscapes on recovery from stress and mental fatigue. Landsc. Urban Plan. 148, 149-158. doi: 10.1016/j.landurbplan.2015.12.015

Liszio, S., Graf, L., and Masuch, M. (2018). The relaxing effect of virtual nature: Immersive technology provides relief in acute stress situations. Ann. Rev. Cyberther. Telemed. 16, 87-93.

Marselle, M. R., Irvine, K. N., and Warber, S. L. (2014). Examining group walks in nature and multiple aspects of well-being: a large-scale study. Ecopsychology 6, 134-147. doi: 10.1089/eco.2014.0027

Mattila, O., Korhonen, A., Pöyry, E., Hauru, K., Holopainen, J., and Parvinen, P. (2020). Restoration in a virtual reality forest environment. Comp. Hum. Behav. 107:106295. doi: 10.1016/j.chb.2020.106295

Mayer, F. S., Frantz, C. M., Bruehlman-Senecal, E., and Dolliver, K. (2009). Why is nature beneficial? The role of connectedness to nature. Environ. Behav. 41, 607-643. doi: 10.1177/0013916508319745

McMahan, R. P., Lai, C., and Pal, S. K. (2016). "Interaction fidelity: the uncanny valley of virtual reality interactions," in Proceedings of the International Conference on Virtual, Augmented and Mixed Reality, (Springer), 59-70. doi: 10.1007/978-3-319-39907-2_6

Ohly, H., White, M. P., Wheeler, B. W., Bethel, A., Ukoumunne, O. C., Nikolaou, V., et al. (2016). Attention restoration theory: a systematic review of the attention restoration potential of exposure to natural environments. J. Toxicol. Environ. Health 19, 305-343. doi: 10.1080/10937404.2016.1196155

Pretty, J., Griffin, M., Peacock, J., Hine, R., Sellens, M., and South, N. (2005a). A countryside for health and wellbeing: The physical and mental health benefits of green exercise - Executive summary. Countryside Recr. 13, 2-7.

Pretty, J., Peacock, J., Sellens, M., and Griffin, M. (2005b). The mental and physical health out- comes of green exercise. Int. J. Environ. Health Res. 15, 319-337. doi: $10.1080 / 09603120500155963$
Pritchard, A., Richardson, M., Sheffield, D., and McEwan, K. (2020). The relationship between nature connectedness and eudaimonic well-being: a meta-analysis. J. Happiness Stud. 21, 1145-1167. doi: 10.1007/s10902-019-0 0118-6

Rockstroh, C., Blum, J., and Göritz, A. S. (2019). Virtual reality in the application of heart rate variability biofeedback. Int. J. Hum. Comp. Stud. 130, 209-220. doi: 10.1016/j.ijhcs.2019.06.011

Rockstroh, C., Blum, J., and Göritz, A. S. (2020). Combining VR and biofeedback: The effects on presence and perceived restorativeness. J. Media Psychol. 32, 176-86. doi: 10.1027/1864-1105/a0 00270

Roe, J., and Aspinall, P. (2011). The restorative benefits of walking in urban and rural settings in adults with good and poor mental health. Health Place 17, 103-113. doi: 10.1016/j.healthplace.2010.09.003

Sakuragi, S., and Sugiyama, Y. (2006). Effects of daily walking on subjective symptoms, mood and autonomic nervous function. J. Physiol. Anthropol. 25, 281-289. doi: 10.2114/jpa2.25.281

Saredakis, D., Szpak, A., Birckhead, B., Keage, H. A. D., Rizzo, A., and Loetscher, T. (2020). Factors associated with virtual reality sickness in head-mounted displays: a systematic review and meta-analysis. Front. Hum. Neurosci. 14:96. doi: 10.3389/fnhum.2020.00096

Schubert, T., Friedmann, F., and Regenbrecht, H. (2001). The experience of presence: factor analytic insights. Presence 10, 266-281. doi: $10.1162 / 105474601300343603$

Slater, M., and Wilbur, S. (1997). A framework for immersive virtual environments (FIVE): Speculations on the role of presence in virtual environments. Presence 6, 603-616. doi: 10.1162/pres.1997.6.6.603

Suma, E. A., Lipps, Z., Finkelstein, S., Krum, D. M., and Bolas, M. (2012). Impossible spaces: Maximizing natural walking in virtual environments with self-overlapping architecture. IEEE Transac. Visual. Comp. Graph. 18, 555-564. doi: 10.1109/TVCG.2012.47

Thompson Coon, J., Boddy, K., Stein, K., Whear, R., Barton, J., and Depledge, M. H. (2011). Does participating in physical activity in outdoor natural environments have a greater effect on physical and mental wellbeing than physical activity indoors? A systematic review. Environ. Sci. Technol. 45, 1761-1772. doi: 10.1021/es102947t

Tinwell, A. (2014). The Uncanny Valley in Games and Animation. New York, NY: CRC Press.

Vasylevska, K., Kaufmann, H., Bolas, M., and Suma, E. A. (2013). "Flexible spaces: dynamic layout generation for infinite walking in virtual environments," in Proceedings of the 2013 IEEE Symposium on 3D User Interfaces (3DUI). (IEEE), 39-42. doi: 10.1109/3DUI.2013.65 50194

Villani, D., and Riva, G. (2012). Does interactive media enhance the management of stress? Suggestions from a controlled study. Cyberpsychol. Behav. Soc. Netw. 15, 24-30. doi: 10.1089/cyber.2011. 0141

Watson, D., Clark, L. A., and Tellegen, A. (1988). Development and validation of brief measures of positive and negative affect: the PANAS scales. J. Pers. Soc. Psychol. 54, 1063-1070. doi: 10.1037/0022-3514.54.6. 1063

Conflict of Interest: The authors declare that the research was conducted in the absence of any commercial or financial relationships that could be construed as a potential conflict of interest.

Copyright (C) 2020 Rockstroh, Blum, Hardt and Göritz. This is an open-access article distributed under the terms of the Creative Commons Attribution License (CC BY). The use, distribution or reproduction in other forums is permitted, provided the original author(s) and the copyright owner(s) are credited and that the original publication in this journal is cited, in accordance with accepted academic practice. No use, distribution or reproduction is permitted which does not comply with these terms. 